\title{
Conventional Total Knee Arthroplasty After Unicondylar Femoral Osteoarticular Allograft Reconstruction: A Case Report
}

\author{
Mahmoud Jabalameli, ${ }^{1}$ Abolfazl Bagherifard, ${ }^{1}$ Hosseinali Hadi, ${ }^{1}$ and Salman Ghaffari ${ }^{1,}{ }^{*}$ \\ ${ }^{1}$ Bone and Joint Reconstruction Research Center, Shafa Orthopedic Hospital, Iran University of Medical Sciences, Tehran, IR Iran \\ "Corresponding author: Salman Ghaffari, Bone and Joint Reconstruction Research Center, Shafa Orthopedic Hospital, Iran University of Medical Sciences, Tehran, IR Iran. Tel: \\ +98-9122882894, Fax: +98-2133542022, E-mail: orthosalman@yahoo.com
}

Received 2017 May 11; Revised 2017 July 03; Accepted 2017 July 06.

\begin{abstract}
Introduction: Osteoarticular allograft, for reconstruction of large bone loss, was introduced for bone loss after tumor resection and subsequently has been used for posttraumatic cases. A main advantage of unicondylar osteoarticular allograft reconstruction is the bone stock preservation, after tumor resection, or traumatic bone loss, providing an opportunity for easier salvage procedures with conventional total knee prosthesis in cases that are complicated by joint pain as well as arthritis.

Case Presentation: A 50-year-old female with post-traumatic medial femoral condyle non-union and bone loss after multiple operations was treated by unicondylar fresh osteochondral allograft reconstruction. Three years later, due to severe pain, progressive varus deformity with varus thrust, as well as osteoarthritis, a total knee arthroplasty with conventional PS prosthesis was performed. A long term follow up reveals a stable prosthesis and acceptable functional outcomes.

Conclusions: Unicondylar osteoarticular allograft reconstruction can be recommended for post-traumatic massive femoral condylar defect. It has relatively good clinical and radiographic results, low rate of complications, and preserves bone stock for future total knee arthroplasty.
\end{abstract}

Keywords: Bone loss, Total Knee Arthroplasty, Unicondylar Osteoarticular Allograft

\section{Introduction}

Structural allograft has many advantages as easy remodeling and filling large segmental defects, excellent biocompatibility, bone stock restoration, and possibility of ligamentous reattachment. Clinical use of osteoarticular allografts for reconstruction of large bone loss was introduced for bone loss after tumor resection (1-3). Long-term results of these reconstructions have been reported to be encouraging. One important advantage of unicondylar osteoarticular allograft reconstructions is the bone stock preservation, allowing for easier salvage procedures with conventional total knee prosthesis in cases of joint pain and arthritis (3-6).

\section{Case Presentation}

A 50-year-old female suffered from a car to pedestrian accident in 2006. She had bilateral distal femoral fracture treated by open reduction and screw fixation in right side and plate and screw fixation in the left side. Due to the left medial femoral condyle nonunion, multiple procedures were performed in another center. Five years after index injury she was referred to our hospital (Figure 1).

Non-united medial condyle with poor bone stock and partial resorption was replaced with fresh unicondylar os-
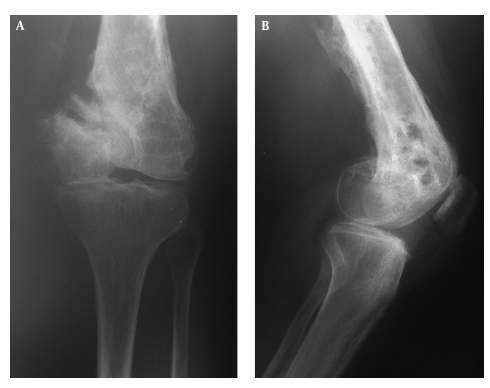

Figure 1. A, Anteroposterior; B, Lateral Radiographs of Left Knee, Showing Nonunion of Medial Femoral Condyle with Bone Loss.

teoarticular allograft and fixed by plate and screws. No intraoperative or postoperative complications occurred. Union achieved about 6 months after surgery. Three years later, due to severe pain especially in patellofemoral compartment, progressive varus deformity with varus thrust as well as osteoarthritis (Figures 2A, 2B, and 3) total knee arthroplasty (TKA) with conventional PS prosthesis (Zimmer-Biomet, Warsaw, Indiana, USA) was performed without any complications.

At the last follow-up in 2017 the prosthesis was stable and painless with 0 - 120 degrees range of motion (Figures $4 \mathrm{~A}$ and $4 \mathrm{~B}$ ). According to the knee injury and osteoarthri- 


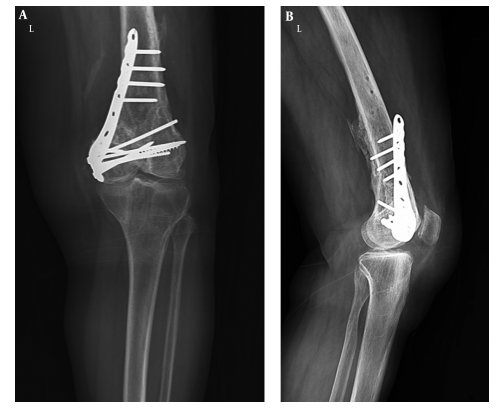

Figure 2. A, Anteroposterior; B, Lateral Radiographs of Left Knee Showing Solid Union of Unicondylar Osteoarticular Allograft.

Figure 3. Weight-Bearing, Hip-To-Ankle Radiograph

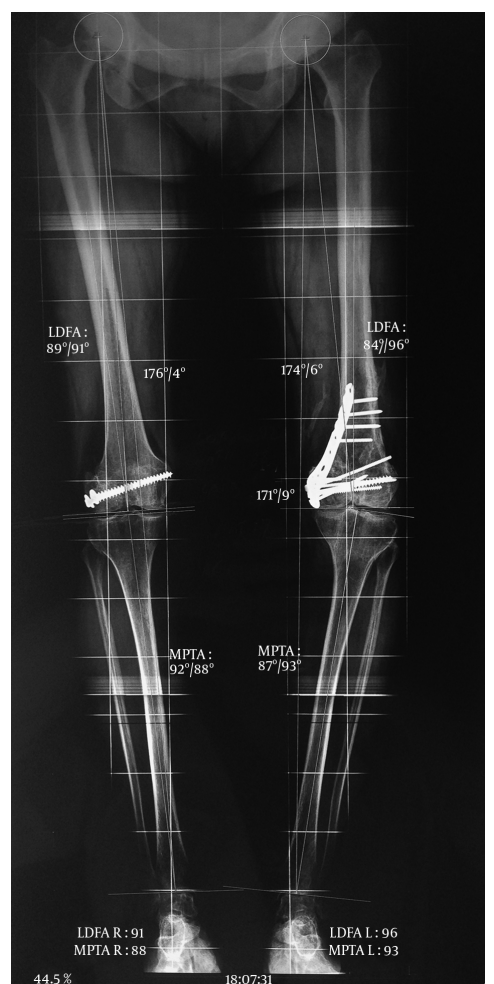

Knee osteoarthritis with varus deformity is evident

tis outcome score (KOOS) (7), the functional result was excellent. This study was approved by the Ethics Committee of our center and our patient gave her written consent to publish the report.

\section{Discussion}

Treatment options for patients with traumatic distal femoral defects include knee arthrodesis, prosthetic joint

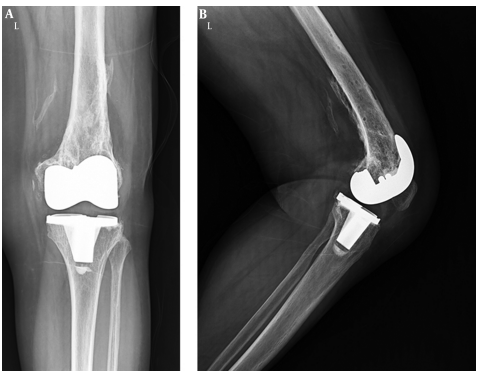

Figure 4. A, Anteroposterior; B, Lateral Radiographs of Left Knee at the Last Follow Up; the Total Knee Prosthesis is Stable.

replacement, osteoarticular allograft reconstruction and amputation (1). When the massive osteoarticular bone loss is limited to one condyle, like our patient, prosthetic reconstruction sacrifices the intact articular surface of the unaffected condyle, leading to unnecessary bone loss (4). Total condylar osteoarticular allografts have been reported to successfully restore the function after tumor resections or post-traumatic large bone defects $(5,7)$. However, little has been published regarding the use of unicondylar osteoarticular allograft reconstruction around the knee after a traumatic injury (8-10).

Campanacci et al. first reported reconstruction of the distal femur with a patellar autograft after a unicondylar resection (1).

Massive allograft application for joint reconstruction is a challenging technique and should be considered as a salvage procedure for severe damage that cannot be managed by more conventional methods (5). Complications of osteoarticular allograft reconstruction are fracture, nonunion, infection, allograft resorption, articular collapse, and joint degeneration (10).

We used fresh osteochondral allograft for reconstruction. Borade et al. described osteoarticular allograft reconstruction in a 4 year-old girl with skeletal defect subsequent to a lawnmower rollover injury (3). Jaffe et al. reporting 8 osteoarticular grafts, 6 hemicondylar, and 2 total condylar, emphasized its value in traumatic articular defects, especially in young patients (4). Muscolo et al. recommended large unicondylar osteoarticular allograft application when post-traumatic bone loss is limited to 1 condyle of the femur or the tibia (5). Lee et al. reported unicondylar osteoarticular reconstruction with an allogenic fresh-frozen condyle in a 45-year-old man with favorable clinical outcome (8).

Levy et al. evaluated 122 patients (129 knees) who underwent osteochondral allograft transplantation of the femoral condyle with graft survivorship of $82 \%$ at 10 years (9). Our patient was able to do daily living activities by her- 
self for about 3 years.

Bianchi et al. evaluated the outcome of 10 distal femoral unicondylar reconstructions with deep frozen allograft. In 1 patient with severe degeneration, pain, and stiffness, like our patient, TKA was performed with conventional total knee prosthesis (10). Morag et al. evaluated 33 patients and 35 knees underwent total knee arthroplasty after a previous tibial or femoral fresh osteochondral allograft implantation. Due to bone stock restoration, the need for augmentation or bone graft was minimal (11). Peters et al. reported reconstruction of the medial femoral condyle and medial collateral ligament in TKA using an Achilles tendon allograft with a calcaneal bone block in 2 young and high-demand patients that obviated the need for a linked or hinged prosthesis (12). Due to bone stock preservation in our case, we performed total knee replacement with conventional PS prosthesis without metal augmentation.

Based on this case and previous publications unicondylar osteoarticular allograft reconstruction can be recommended for post-traumatic massive femoral condylar defect. It has relatively good clinical and radiographic results, low rate of complications, and preserves bone stock for future TKA.

\section{Footnotes}

Authors Contribution: All the authors contributed to this case report. Study design: Mahmoud Jabalameli, Abolfazl Bagherifard; patients treatment and follow up: Mahmoud Jabalameli, Abolfazl Bagherifard, Hosseinali Hadi, Salman Ghaffari; drafting and critical revision of the manuscript: Mahmoud Jabalameli, Abolfazl Bagherifard, Hosseinali Hadi, Salman Ghaffari; study supervision: Mahmoud Jabalameli.

Conflict of Interest: The authors declared no conflict of interest.

\section{References}

1. Campanacci M, Cervellati C, Donati U. Autogenous patella as replacement for a resected femoral or tibial condyle. A report on 19 cases. $J$ Bone Joint Surg Br. 1985;67(4):557-63. [PubMed: 4030850].

2. Gross AE, Kim W, Las Heras F, Backstein D, Safir O, Pritzker KP. Fresh osteochondral allografts for posttraumatic knee defects: long-term followup. Clin Orthop Relat Res. 2008;466(8):1863-70. doi:10.1007/s11999008-0282-8. [PubMed:18465182].

3. Borade A, Kempegowda H, Fernandez M, Horwitz DS. Osteoarticular allograft reconstruction of post-traumatic defect of distal femur in a pediatric patient: A case report and literature review. Injury. 2016;47(11):2473-8. doi: 10.1016/j.injury.2016.09.015. [PubMed: 27638000].

4. Jaffe KA, Morris SG, Sorrell RG, Gebhardt MC, Mankin HJ. Massive bone allografts for traumatic skeletal defects. South Med J. 1991;84(8):97582. [PubMed: 1882275].

5. Muscolo DL, Ayerza MA, Aponte-Tinao LA, Abalo E, Farfalli G. Unicondylar osteoarticular allografts of the knee. J Bone Joint Surg Am. 2007;89(10):2137-42. doi: 10.2106/JBJS.F.01277. [PubMed: 17908888].

6. Hornstein S, Moukoko D, Deroussen F, Plancq MC, Collet LM, Gouron R. Successful hemicondylar femoral allograft for traumatic bone loss: a paediatric case study with ten years of follow-up. Knee. 2015;22(1):636. doi: 10.1016/j.knee.2014.10.008. [PubMed: 25467933].

7. KOOS . Knee injury and Osteoarthritis Outcome Score(KOOS) 2014. Available from: www.koos.nu.

8. Lee JH, Wang SI, Choi HR, Hwang BY, Lim YJ, Wie JS, et al. Unicondylar osteoarticular allograft reconstruction of the distal femur in a patient with a traumatic osteoaticular defect of the lateral femoral condyle. Knee Surg Sports Traumatol Arthrosc. 2011;19(4):556-8. doi: 10.1007/s00167-010-1252-8. [PubMed: 20811875].

9. Levy YD, Gortz S, Pulido PA, McCauley JC, Bugbee WD. Do fresh osteochondral allografts successfully treat femoral condyle lesions? Clin Orthop Relat Res. 2013;471(1):231-7. doi: 10.1007/s11999-012-2556-4. [PubMed: 22961315].

10. Bianchi G, Staals EL, Donati D, Mercuri M. The use of unicondylar osteoarticular allografts in reconstructions around the knee. Knee. 2009;16(1):1-5. doi:10.1016/j.knee.2008.07.011. [PubMed: 18954989].

11. Morag G, Kulidjian A, Zalzal P, Shasha N, Gross AE, Backstein D. Total knee replacement in previous recipients of fresh osteochondral allograft transplants. J Bone Joint Surg Am. 2006;88(3):541-6. doi: 10.2106/JBJS.D.02816. [PubMed: 16510820].

12. Peters CL, Dienst M, Erickson J. Reconstruction of the medial femoral condyle and medial collateral ligament in total knee arthroplasty using tendoachilles allograft with a calcaneal bone block. J Arthroplasty. 2004;19(7):935-40. [PubMed: 15483814]. 